\title{
Endothelium-modulated proliferation of medial smooth muscle cells: influence of angiotensin II and converting enzyme inhibition
}

\author{
A. W. A. HAHN, R. SCHMidT*, F. KERN, T. J. Resink AND F. R. BÜHLER \\ Department of Research, University Clinics Basel, CH 4031 Basel, Switzerland and \\ ${ }^{*}$ Hoffmann La Roche Ltd., CH 4002 Basel, Switzerland
}

KEY WORDS: Smooth muscle cell, medial proliferation, endothelium, angiotensin II, angiotensin converting enzyme inhibition.

This study investigated the role of the endothelium and angiotensin II (Ang II) in regulating medial smooth muscle cell (SMC) proliferation. ['H]-thymidine incorporation into medial SMC of rat arteries was examined in vivo, using ballooned rat carotid arteries, as well as in vitro, using cultures of aortic tissue rings (organoids). In vivo, maximal medial $\left.{ }^{\beta} \mathrm{H}\right]-$ thymidine incorporation occurred within 3 days post-ballooning. In endothelium-denuded organoids, maximum medial DNA synthesis was achieved after 7 days of culture. [3H]-thymidine-labelling of SMC in intact organoids (with endothelium) increased minimally during culture, indicating that the endothelium provided protection with respect to medial proliferation under basal conditions (culture in the presence of $1 \%$ plasma-derived serum). Inclusion of $10^{-7} M$ Ang.II significantly elevated medial ${ }^{\beta} \mathrm{HJ}$-thymidine incorporation above that in control cultures. The stimulatory effect of Ang II was much more pronounced in intact organoids than in endothelium-denuded organoids, indicating synergistic growth regulation by Ang II and endothelium-derived factors. When organoids were cultured in the combined presence of Ang II and the ACE inhibitor cilazaprilat, labelling indices of intact organoids were also significantly increased above control, but to a lower level than those obtained in the presence of Ang II alone. However, for endothelium-denuded organoids, medial $\mathrm{PH}$ ]-thymidine incorporation in the combined presence of Ang $I I$ and cilazaprilat was not significantly different from that in untreated controls. Thus, cilazaprilat exerts both endothelium-dependent and endothelium-independent negative regulatory effects on medial SMC proliferation.

\section{Introduction}

Angiotensin converting enzyme inhibition in rat models of restenosis and hypertension has been reported to inhibit age-, pressure- and injury-dependent formation of arterial lesions ${ }^{[1]}$. These effects have been largely attributed to the inhibition of local angiotensin II (Ang II) generation. Studies on vascular smooth muscle cells (SMC) both in vitro and in vivo have demonstrated that Ang II is an important mediator of long-term vascular remodelling ${ }^{[2,3]}$. However, modification of autoregulatory growth responses in SMC by Ang II is not the only regulator of SMC proliferative behaviour. SMC function/differentiation is also subject to control by external trophic substances, such as adrenergic hormones and components of the extracellular matrix ${ }^{[4]}$, as well as the paracrine influence of the endothelium ${ }^{[5]}$. The endothelium releases a variety of substances which affect arterial tone ${ }^{[6]}$ and it is also an important source of factors which exert stimulatory or growth inhibitory influences on SMC proliferative behaviour ${ }^{[6]}$. To better delineate the intrinsic growth regulatory capacity of the vessel wall the present study has investigated medial SMC proliferation both in vivo, after ballooning of the rat carotid artery, as well as in an in vitro model of ringed aortic segments (organoid culture) in

Correspondence. Alfred W. A. Hahn, PhD, Department of Research, ZLF 319, University Clinics Basel, Hebelstrasse 20, CH 4031 Basel, Switzerland. which systemic and nervous influences that exist in vivo are eliminated. The effects of Ang II and cilazapril have also been studied in order to assess whether angiotensin converting enzyme inhibition exerts its growth modulatory properties on the vessel by mechanisms other than mere prevention of local Ang II formation.

\section{Materials and methods}

MATERIALS

Angiotensin II was purchased from Bachem Feinchemikalien AG, Bubendorf, Switzerland. Cilazapril and Cilazaprilat were from Hofmann La Roche Ltd. Basel, Switzerland.

BALLOON-CATHETERIZATION OF CAROTID ARTERIES

Endothelial denudation and vascular injury were achieved in the left common carotid artery of 4-month-old normotensive rats as described ${ }^{[7]}$.

\section{ORGANOID CULTURES}

Twenty-week old rats were obtained from Charles River Wiga, Sulzfeld, Germany. One group of animals $(n=6)$ was treated over a period of 6 days with the ACE inhibitor cilazapril $\left(10 \mathrm{mg} \cdot \mathrm{kg}^{-1} \cdot \mathrm{day}^{-1}\right)$ prior to being killed. The rats were decapitated after anaesthesia, their aortae removed under sterile conditions and cleansed of excessive adventi- 
tia. Aortae were sliced into $\sim 0.5 \mathrm{~cm}$-wide rings, either left intact or mechanically denuded of the endothelium, and cultured as free-floating segments (aortic organoids) in OptiMEM (Gibco/BRL) supplemented with $1 \%$ plasmaderived serum (PDS, prepared as described by Ross and Kariya ${ }^{[7]}$ ) with medium changes every second day. The free floating aortic organoids were maintained for up to at least 14 days without any occurrence of explant culture. Compared with intact aortic rings, de-endothelialized segments did not exhibit relaxation responses to acetylcholine or histamine but did retain a full depolarization-induced contractile response. Organoids were cultured in the absence and continuous presence of $10^{-7} \mathrm{M}$ Ang II and without or with inclusion of $0 \cdot 1 \mathrm{mg} \cdot \mathrm{ml}^{-1}$ cilazaprilat. Ang II was added twice daily throughout the culture period. For $\left[{ }^{3} \mathrm{H}\right]$-thymidine labelling, organoids were incubated with $1 \mu \mathrm{Ci} . \mathrm{ml}^{-1}$ of radiolabelled nucleotide for the last $48 \mathrm{~h}$ of the different culture periods. The minor proliferation response (assessed by cell enumeration as described previously ${ }^{[8]}$ ) of cultured rat aortic smooth muscle cells to $1 \%$ PDS (1.3-fold increase in cell number after 10 days in culture) assured that PDS preparations used were essentially mitogen-free.

\section{HISTOCHEMISTRY}

Organoids were sliced in half and embedded in TissueTek OCT (Miles Laboratories Inc., Naperville, IL, U.S.A.) with the newly cut edges facing upwards. Mounted tissue was frozen rapidly in an isopentane bath in liquid nitrogen. Sections of $\sim 5 \mu \mathrm{m}$ were cut in a freezing microtome, applied to polylysine-coated slides, dried and stored at $-70^{\circ} \mathrm{C}$. Sections were developed and stained in Meyer's hematoxilin.

\section{STATISTICS}

$\mathrm{n}$ values given in Figure legends represent the number of different animals from which organoids were prepared. For every experimental condition at least eight sections of each organoid were analysed. Data were statistically analysed by ANOVA and expressed as means $\pm S D$, and statistically significant differences are indicated in the Figures. The proliferation index was calculated as mean number of labelled cells/total cell population and section.

\section{Results}

The time course of medial SMC proliferation which occurred in vitro following endothelial denudation of aortic organoids was compared with that occurring in vivo after ballooning of carotid arteries. In carotid arteries, maximum medial proliferation was achieved within 3 days post-ballooning and thereafter decreased progressively to reach a baseline proliferation index post-ballooning (Fig. 1). In cultured aortic organoids, medial proliferation reached maximum 7 days after endothelial denudation and then decreased to baseline after 14 days in culture (Fig. 1). A negligible medial proliferative response was observed (labelling indices not significantly different from day 0 ; data not shown) when organoids were cultured for up to 14 days under completely serum-free conditions (i.e. 1\% PDS

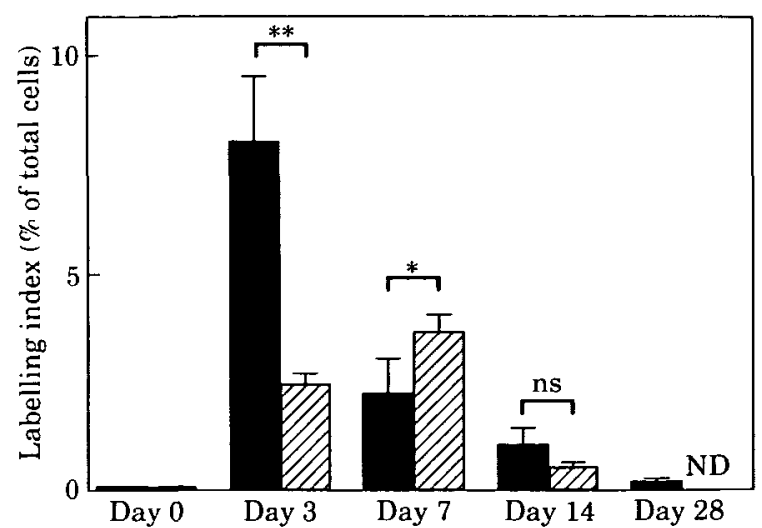

Figure 1 Comparison of $\left[{ }^{3} \mathrm{H}\right]$-thymidine incorporation into medial SMC either in vivo following balloon catheterization of rat carotid arteries (solid bars), or in vitro following endothelialdenudation of aortic tissue rings (hatched bars). Data are given as mean $\pm \mathrm{SD}, \mathrm{n}=10 ; * P \leq 0.05 ; * * P \leq 0.001 ; \mathrm{ns}=$ not significant; $\mathrm{ND}=$ labelling index of organoids after 28 days in culture not determined.

replaced by $1 \%$ bovine serum albumin). In the presence of $1 \%$ PDS the maximal medial SMC proliferation index achieved in de-endothelialized aortic organoids was only $\sim 40 \%$ of that observed in vivo following ballooning of carotid arteries.

To investigate the role of the endothelium in SMC proliferation in aortic organoids, $\left[{ }^{3} \mathrm{H}\right]$-thymidine incorporation into medial SMC of intact and de-endothelialized aortic rings was measured after 3 and 7 days of culture under basal conditions (OptiMEM/1\% PDS). Analysis at day 14 was not routinely included because proliferation indices had returned to baseline by this time (see Fig. 1). In organoids with an intact endothelium the proliferation index did not exceed $1 \%$ at either of the culture periods (Fig. 2). $\left[{ }^{3} \mathrm{H}\right]$-thymidine labelling of medial SMC in endothelium-denuded organoids was $5-6$ fold greater then in intact organoids $(P<0.001$ for proliferation index at

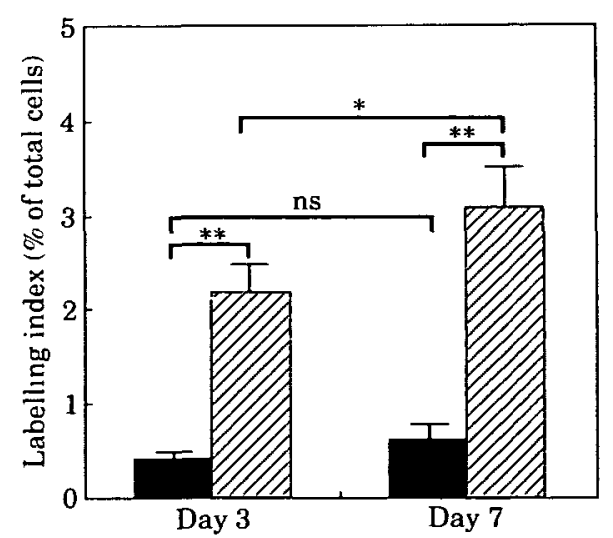

Figure $2\left[{ }^{3} \mathrm{H}\right]$-thymidine incorporation into medial SMC after 3 and 7 days of culture of either intact (solid bars) or endotheliumdenuded aortic organoids (hatched bars). Data are given as mean $\pm \mathrm{SD}, \mathrm{n}=8 ;{ }^{*} P \leq 0.05 ; * * P \leq 0.001 ; \mathrm{ns}=$ not significant. 


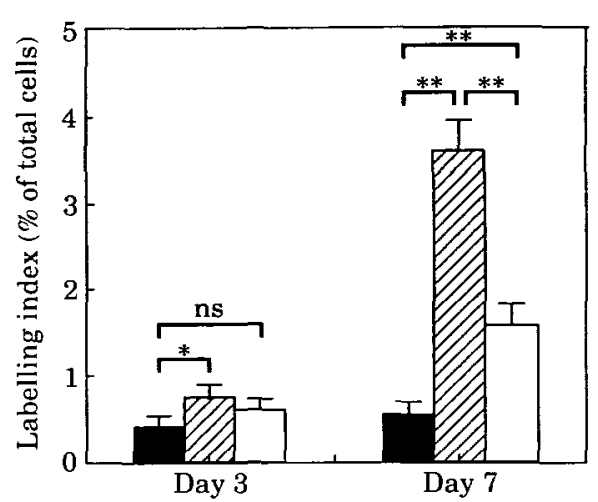

Figure $3\left[{ }^{3} \mathrm{H}\right]$-thymidine incorporation into medial SMC in intact aortic organoids was measured after 3 and 7 days culture under basal conditions ( $1 \%$ PDS; solid bars) or with inclusion of Ang II (hatched bars) or the combination of Ang II and cilazaprilat (open bars). Organoids were obtained from ACE inhibitor-pretreated rats. Data are given as mean $\pm \mathrm{SD}, \mathrm{n}=6 ;{ }^{*} P \leq 0.05 ;{ }^{* *} P \leq 0.001$; ns $=$ not significant

either 3 or 7 days; Fig. 2). Some proliferation of adventitial fibroblasts and also eventually of endothelial cells (day 14) was observed (data not included in this paper).

Figures 3 and 4 present data for medial proliferation in organoids from cilazapril treated animals following culture under control conditions (OptiMEM/1\% PDS) and with inclusion of either $10^{-7} \mathrm{M}$ Ang II or a combination of Ang II and cilazaprilat. Cilazapril treatment of animals per se did not significantly influence basal medial SMC proliferation in either intact or endothelium-denuded rings (compare control values in Figs 3 and 4 with data fom untreated animals in Fig. 2). In intact organoids from cilazapril-treated animals, inclusion of $10^{-2} \mathrm{M}$ Ang II significantly elevated medial proliferation indices compared to unstimulated

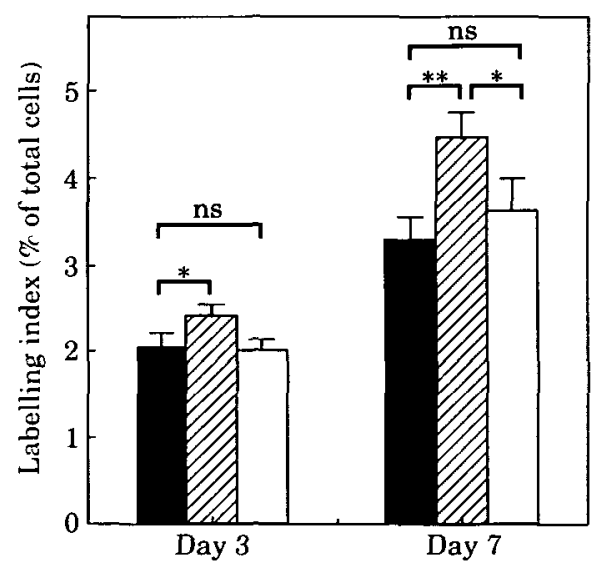

Figure $4\left[{ }^{3} \mathrm{H}\right]$-thymidine incorporation into medial SMC in endothelium-denuded aortic organoids was measured after 3 or 7 days culture under basal conditions ( $1 \%$ PDS; solid bars) or with inclusion of Ang II (hatched bars) or Ang II/cilazaprilat (open bars). Organoids were obtained from $A C E$ inhibitor-pretreated rats. Data are given as mean $\pm \mathrm{SD}, \mathrm{n}=10 ;{ }^{*} P \leq 0.05 ;{ }^{* *} P \leq 0.001$; ns $=$ not significant. controls after both 3 days $(P<0.05)$ and 7 days $(P<0.001)$ in culture (Fig. 3). Ang II also induced $\left[{ }^{3} \mathrm{H}\right]-$ thymidine labelling in endothelium-denuded organoids $(P<0.05$ at day 3 and $P<0.001$ at day 7 ; Fig. 4), although due to the elevated basal proliferation index in these organoids the relative stimulatory effect of Ang II (foldincrease above basal was 0.6 and 1.3 at days 3 and 7 , respectively Fig. 4) was less than in intact organoids (fold-increase above basal was 1.8 and 5.4 at days 3 and 7 , respectively; Fig. 3 ). It is noteworthy that the absolute labelling indices after 7 days culture in the presence of Ang II did not differ significantly between intact $(3.7 \pm 0.4)$ and de-endothelialized $(4 \cdot 4 \pm 0.3)$ tissues. When PDS in culture medium was replaced by $1 \%$ bovine serum albumin the stimulatory effects of Ang II on $\left[{ }^{3} \mathrm{H}\right]$-thymidine labelling in intact and de-endothelialized organoids was not observed (data not shown). For organoids cultured in the combined presence of Ang II and cilazaprilat, medial $\left[{ }^{3} \mathrm{H}\right]$-thymidine labelling was greater than that in control organoids $(P<0.001$ at day 7), but did not achieve that level observed in organoids exposed only to Ang II $(P<0.001$ at day 7$)$ (Fig. 3). In contrast, for de-endothelialized organoids labelling indices in controls and those cultured in the combined presence of Ang II and cilazaprilat were not significantly different (Fig. 4).

\section{Discussion}

The present study has demonstrated that mechanical endothelial denudation of aortic organoids in vitro results in medial SMC proliferation which is comparable, in terms of early onset and transience, to the proliferative response observed in vivo following ballooning of the carotid artery. For the purposes of investigating the autoregulative capacity of a vessel and its modulation by defined external stimuli (e.g. growth modulators or pharmacalogical compounds), our in vitro organoid model is advantageous in that confounding systemic and neuronal influences present in vivo are absent.

The occurrence of SMC medial proliferation in cultured organoids required inclusion of minimal levels of serum (1\% plasma-derived serum) during culture. Additionally, comparison of the $\left[{ }^{3} \mathrm{H}\right]$-thymidine labelling indices in either intact or endothelium-denuded organoids revealed a conspicuous protective effect of the endothelium on medial SMC proliferation. This may indicate that in intact organoids, either medial SMC are relatively (vs endothelium-denuded organoids) inaccessible to PDS-contained growth-promoting factors or that the endothelium might constitutively release negative-growth regulatory factors which counter the mitogenic activities contained in PDS. However, PDS is an essentially mitogen-free preparation of serum $^{[7]}$, as demonstrated by its minimal support of the growth of isolated SMC in culture (methods and refs. ${ }^{[7,8]}$ ). In vivo it has been shown that after endothelial denudation of the carotid artery by ballooning, metalloproteases within the media are activated ${ }^{[9]}$ with resultant depolymerization of the extracellular matrix, proteolytic degradation of extracellular matrix constituents and the release of bio- 
logically active degradation products (e.g. EGF-like peptides) which can synergistically support SMC proliferation $^{[10]}$. It is thus possible that the mitogenic activity of PDS in endothelium-denuded organoid cultures may also arise from medial SMC-induced activation of PDS-containing latent growth-promoting substances. Mesenchymal proliferation is unlikely to be due to an unspecific wound-healing response to endotheliumdenudation since negligibile mesenchymal proliferation was detected in unstimulated controls.

The protective effect of the endothelium was also evident when organoids derived from cilazapril-treated animals were cultured under conditions of continuous in vitro angiotensin converting enzyme inhibition, indicating that the negative growth-modulatory effect of the endothelium is not linked to the local renin-angiotensin system. Moreover, the stimulatory effect of Ang II on SMC proliferation (i.e. the several-fold-increase of labelling above control proliferation indices) was greatest in intact organoids. This further supports the argument that the protective effect of the endothelium does not relate to inhibition of the effects of Ang II. In vivo, Ang II has been demonstrated to act as a long-term mitogen ${ }^{[11]}$, an effect proposed to involve co-mediatory effects of the $\beta$-adrenergic system ${ }^{[11]}$. Our findings that Ang II stimulates medial SMC proliferation in cultures of aortic organoids, where co-participation of the $\beta$-adrenergic system is absent, would, however, indicate that the growth promoting activity of Ang II is independent of the nervous system.

There was no medial SMC proliferation response to Ang II in either intact or endothelium-denuded organoids under completely serum-free culture conditions indicating a requirement for some co-mitogenic factor(s) present in plasma-derived serum. The effect of Ang II on SMC proliferation in organoids not only requires the presence of PDS but was also greatest in intact organoids. These data suggest that either PDS stimulates the release of endothelium-derived factors, which, per se, are minimally effective in promoting SMC growth but which can synergize with Ang II, or that Ang II itself promotes release of co-mitogenic factors from the endothelium. Since endotheliumdenuded organoids exhibited a significant SMC growth response to Ang II, the ability of Ang II to promote medial SMC proliferation is, however, not necessarily dependent on endothelium-derived growth promoting factors.

The proliferative response of organoids cultured in the combined presence of Ang II and cilazaprilat was weaker than that of organoids exposed to Ang II alone, and this difference was highly significant in intact organoids. Since the presence of cilazaprilat during culture cannot significantly alter the effective concentrations of Ang II $\left(10^{-7} \mathrm{M}\right.$ already present) the blunted proliferative response of Ang II/cilazaprilat (vs Ang II alone) in cultured organoids may reflect an action of cilazapril that is unrelated to inhibition of angiotensin converting enzyme. The contribution of elevated tissue bradykinin to this phenomenon can be excluded since exogenously added bradykinin did not mimic Ang II-mediated effects (labelling index less than 1) and neither was Ang II-stimulated mitogenesis inhibited by bradykinin antagonist (labelling index $\approx 5$ ). Since the negative effect of cilazaprilat on medial SMC proliferation is more pronounced in intact than endothelium-denuded organoids, an endothelium-dependent mode of action, possibly the release of growth-inhibitory substances for this compound, is implied. Importantly, although Ang II stimulates medial SMC proliferation in endothelium-denuded organoids, this proliferation response is no longer manifest in organoids cultured in the combined presence of Ang II and cilazaprilat. Thus cilazaprilat also exerts endotheliumindependent effects. It is not yet known whether these effects related to inhibition of metalloproteases other than angiotensin converting enzyme (e.g. medial SMC enzymes which activate latent serum or extracellular matrix factors).

The data herein illustrate the complex and powerful regulation of medial SMC proliferation by the endothelium. Several candidate categories of endothelium-derived substances such as NO, prostanoids, and peptides are likely to be involved. The culture of aortic organoids should provide a useful model for investigating the growth-regulatory potential of specific endothelium-derived substances, as well as their interactions with one another.

This study was supported by Swiss National Foundation grant No. 31-35732.92; Sandoz Foundation and Ciba-Geigy Jubilaeums Stiftung.

\section{References}

[1] Powell JS, Clozel J-P, Müller RKM et al. Inhıbitors of angiotensin converting enzyme prevents myointimal thickening after vascular injury. Science $1989 ; 245$ : 186-8.

[2] Schelling P, Fischer H, Ganten D. Angiotensin and cell growth: a link to cardiovascular hypertrophy? J Hypertens 1991; 9: 3-15.

[3] Hahn AWA, Kern F, Buhler FR, Resink TJ. The reninangiotensin system and extracellular matrix. Clin Invest $1983 ; 71: 7-12$.

[4] Hedin U, Bottger BA, Forsberg E, Johannson S, Thyberg J. Diverse effects of fibronectin and laminin on phenotypic properties of cultured arterial smooth muscle cells. J Cell Biol 1988; 107: 307-19.

[5] Dzau VJ, Gibbons GH, Pratt RE. Molecular mechanisms of vascular renin-angiotensin system in myointimal hyperplasia. Hypertension 1990; 18: 100-5.

[6] Luischer TF, Vanhoutte PM. The Endothelium-Modulator of cardiovascular function. Boca Raton: CRC Press 1990: $1-215$.

[7] Ross R, Kariya B. Morphogenesis of vascular smooth muscle in atherosclerosis and cell culture. In: Bohr, D, Somlyo, AP, Sparks, HV Jr, eds. Handbook of Physiology, Sect. 2: The Cardiovascular System, Vol. 2. Washington, DC: American Physiological Society, 1980: 69-75.

[8] Resink TJ, Scott-Burden T, Hahn AWA et al. Specific growth stimulation of cultured smooth muscle cells from spontaneously hypertensive rats by platelet-derived growth factor A-chain homodimer. Cell Reg 1990; 1: 821-31.

[9] Birkedal-Hansen H, Moore WG, Bodden MK, Engler JA. Matrix metalloproteinases: a review. Crit Rev Oral Biol Med $1993 ; 4: 197-250$.

[10] Engel J. EGF-like domains in extracellular matrix proteins: localized signals for growth and differentiation. FEBS Lett 1989; 251: 1-7.

[11] Raguki H, Jacob HJ, Krieger JE, Ingelfinger JR, Pratt RE. Vascular injury induces angiotensinogen gene expression in the media and neointima. Circulation 1993; 87: 883-90. 